REVISTA DE DERECHO UNED, NÚM. 13, 2013

\title{
ANÁLISIS HISTÓRICO DE LA DESIGUALDAD POR RAZÓN DE GÉNERO EN MÉXICO
}

\author{
«HISTORICAL ANALYSIS OF INEQUALITY BECAUSE OF \\ GENDER IN MEXICO»
}

MINERVA E. MARTÍNEZ GARZA

Licenciada en Derecho y Maestra en Ciencias Penales

Presidenta de la Comisión Estatal de Derechos Humanos

del Estado de Nuevo León, México

Resumen: La historia es una herramienta que nos permite dimensionar el papel que han desempeñado hombres y mujeres a lo largo de las diversas etapas del desarrollo de una nación, y la historia de las mujeres nos permite visualizar las dificultades que han tenido que afrontar en la búsqueda de su reconocimiento como personas con derechos, a fin de alcanzar la igualdad real o material y ante la ley, las autoridades y la propia sociedad. Bajo esa perspectiva, se plasman en este artículo los acontecimientos prósperos y adversos que han vivido las mujeres en el contexto de una sociedad persistentemente androcéntrica, desde sus orígenes en la época precortesiana, pasando por el periodo colonial, el del movimiento independentista, los inicios del siglo XX y el posterior a la proclamación de la ley fundamental, hasta los albores del tercer milenio, en el que algunos datos estadísticos confirman que la igualdad entre mujeres y hombres sigue siendo una asignatura pendiente.

Palabras clave: Mujeres, discriminación, desigualdad de género, historia, derechos, violencia, épocas, datos estadísticos, México.

Abstract: History is a tool that allows us to gauge the role played by men and women along the different stages of development of a nation, and women's history allows us to see the difficulties that have 
been faced in seeking recognition as persons with rights, in order to achieve real and material equality under the law, the autorithies and the society. By itself under this perspective, is reflected in this article prosperous and adverse events that women have experienced in the context of a persistently androcentric society, from its origins in pre-Conquest times, going through the colonial period, the independence movement, early years of the twentieth century and after the proclamation of the fundamental law, until the dawn of the third millennium, in some statistical data confirm that equality between women and men remains a pending matter.

Keywords: Women, discrimination, gender inequality, history, rights, violence, epoch, statistical data, Mexico.

Recepción original: 12/09/2013

Aceptación original: 25/09/2013

\section{INTRODUCCIÓN}

La documentación de fechas, eventos, datos y acontecimientos violentos que ha vivido la humanidad, esto es, la historia misma de la civilización humana, tiene una función útil para la identificación y solución de problemas, es precisamente esa la función herramental de la historia; sin embargo, hay que tomar en consideración las situaciones desfavorables que pudiera presentar, ya que los anales históricos son elaborados, la mayoría de las veces, por los vencedores, derivando lo anterior en una parcialidad al momento de estudiarla. Lo anterior puede resultar en un desconocimiento de la visión de sociedades, grupos o individuos marginados o en situación de marginación ${ }^{1}$. De ahí la dificultad de documentar y hacer un esbozo de marco histórico para situar a las y los lectores dentro de la problemática que plantea la desigualdad de género, específicamente en México.

A pesar del problema que presenta referenciar los eventos del pasado, es importante hacerlo, toda vez que se ha producido un progresivo cambio en la percepción de los derechos del género femenino, por parte de hombres y mujeres; esta percepción se basa precisamente en el conocimiento de aquellos cambios coyunturales y paradigmáticos, que pasan a ser eventos relevantes en la historia de la huma-

1 El presente envuelve al pasado y en el pasado toda la historia la han hecho los varones... «todo cuanto sobre las mujeres han escrito los hombres debe tenerse por sospechoso, puesto que son juez y parte a la vez». BEAUVOIR, Simone D', «El Segundo Sexo», Ed. Siglo Veinte, México, 2005, p. 7. 
nidad, aún y cuando en la práctica subsisten y persisten infinidad de obstáculos, lo anterior lo sabemos porque lo hemos visto de cerca y podemos percibir que aún falta un largo camino por recorrer ${ }^{2}$.

Se presenta a continuación un breve repaso de la trayectoria histórica de las mujeres en México, lo que permitirá identificar evidencias de la discriminación que la sociedad androcéntrica les ha inferido, así como los antecedentes de la lucha que han seguido para lograr la reivindicación de sus derechos.

Para comprender la situación actual de las mujeres en México, es conveniente hacer un repaso del rol que han jugado a lo largo de la historia, haciendo un recorrido desde la época precolombina hasta los albores del tercer milenio.

\section{1. Época Prehispánica}

Entre los aztecas, las mujeres constituían la piedra angular de la sociedad ya que todas las actividades se desarrollaban en torno a la familia, y eran el motor de ésta, por lo que su importancia radicaba, más que en el estatus de autoridad reconocida, en su carácter de indispensable para el funcionamiento de la vida cotidiana.

Aparentemente es un hecho que la mujer azteca estaba subordinada respecto al hombre. Eran educadas para servir, adorar, respetar y obedecer a su marido, debían ser pasivas y sumisas, y no tomaban decisiones sobre su propia vida, máxime su pertenencia a la clase alta.

La mujer noble, de la clase de los pillis, compartía con el hombre noble privilegios como la posibilidad de heredar tierras. Al mismo tiempo, le era exigido mantenerse virgen hasta el matrimonio, debía ser obediente, recatada y honrada, Mientras era soltera, la mujer permanecía bajo la custodia del padre, y al casarse pasaba a estar bajo la autoridad del marido. Mientras el hombre podía no ser casto previo al matrimonio, a la mujer se le exigía inclusive la fidelidad aún después de muerto el marido. Algunas doncellas podían ingresar al servicio en el templo, siempre excluidas de los privilegios del sacerdocio.

2 Ahora bien, la mujer siempre ha sido, si no la esclava del hombre, al menos su vasalla; los dos sexos jamás han compartido el mundo en pie de igualdad; y todavía hoy, aunque su situación está evolucionando, la mujer tropieza con graves desventajas... Incluso cuando se le reconocen en abstracto algunos derechos, una larga costumbre impide que encuentre en los usos corrientes su expresión completa. Ídem. 
La mujer de los macehuallis (hombres libres), debía pagar tributo tal y como lo hacía el hombre de su clase. Su participación económica no se limitaba a las labores agrícolas, sino al trabajo de hilado y costura necesario para vestir a su familia, además del trabajo como doméstica en las casas señoriales. También se le exigían recato, obediencia y honestidad, pero las exigencias sexuales no eran equivalentes a las de la clase dominante; la prostitución, por ejemplo, les era permitida y en algunos casos hasta impuesta. El estrato más bajo en la escala social, lo ocupaban las esclavas, las que caían en este rango por deudas, por captura de guerra o por voluntad propia o familiar. Los esclavos en general eran tratados como propiedad del amo, mercancía que podía ser vendida, rentada o destruida por éste ${ }^{3}$.

\section{2. Época Colonial}

La llegada de los españoles jugó un papel fundamental en la interacción de los géneros en México, dando inicio a la época colonial. A la discriminación ya existente tanto en la sociedad española como en la indígena, se sumó el factor de que los conquistadores, los dominadores, los poderosos, eran hombres.

La leyenda contaba que Quetzalcóatl, el dios de las barbas y piel blanca, regresaría por el mar desde el oriente. Por lo que los indígenas, creyendo que los españoles eran los dioses que cumplían la promesa, los cubrieron de presentes. Como el sacrificio de doncellas a los dioses era una práctica común y honorable, los españoles reci-

3 La educación de la niña estaba encomendada a la madre... la niña aprendía a tejer el algodón, y a hilar un año después... la educación de la niña no era menos cuidadosa, desde los 12 años era considerada mayor y se levantaba a media noche para barrer y arreglar la casa. Tenía la obligación de no desnudarse para descansar, tanto para que fuera recatada, cuanto para que estuviera lista para acudir al llamado de la madre inmediatamente; a los 13 años era corriente que supiera no sólo hilar y tejer sino, en general, estaba apta para dirigir una casa... las mujeres no salían solas ni acostumbraban marchar al lado del marido; en las comidas se mantenían en silencio... El matrimonio se efectuaba a edad temprana, los varones a los 20 y las muchachas a los $16 \ldots$ era obligatorio contraer matrimonio, pero sólo era bien visto el monógamo... había causa de divorcio, tanto si la mujer era estéril, cuanto si el hombre no subvenía a las necesidades del hogar... el hombre podía ser polígamo, pero siempre era preferida la primera mujer sobre las subsecuentes. La mujer sólo tenía un esposo... así mismo se permitía una o varias concubinas, siempre que fueran solteras... existía también la escuela para sacerdotisas, donde además aprendían el arte plumario y el del tejido... Entre los purépechas, cuando un rey moría, sus mujeres eran sacrificadas y enterradas con él, pero el cuerpo del monarca era incinerado. LOZANO FUENTES, José Manuel, LOPEZ REYES, Amalia, "Historia General de México», 2. ${ }^{a}$ ed., Ed. Publicaciones Cultural, s. l. n. f., pp.59-60, 71. 
bieron como tributo, además de oro y plata, a mujeres indígenas. El mestizaje, por otra parte, contribuyó a la formación del estereotipo del vencedor, para el hombre, y el vencido para las mujeres ${ }^{4}$.

Trescientos años de la colonia terminaron cuando el pueblo decidió conspirar y organizarse para iniciar la Guerra de la Independencia mexicana, en la que existieron grandes mujeres que fueron determinantes para dicha causa, distinguiéndose entre ellas: Doña Josefa Ortiz de Domínguez, Gertrudis Bocanegra y Leona Vicario; quienes, haciendo caso omiso de los prejuicios de la época, se unieron a la lucha por la libertad de nuestro país.

Por compartir unos datos, no debemos olvidar que durante el inicio de la guerra, Leona Vicario se convirtió en informante de los insurgentes sobre los acontecimientos de la capital; la cual se comunicó mediante informes en clave publicados en el periódico $E l$ Ilustrador Americano. Contribuyó a la lucha con más de $\$ 80,000.00$ pesos plata y, hecha prisionera, se negó a descubrir a sus «cómplices» y una vez liberada, colaboró con Morelos ${ }^{5}$.

Mientras que Gertrudis Bocanegra, la «heroína de Pátzcuaro», durante la guerra de Independencia, se estableció en una casa en su ciudad natal, en la que desarrolló desde el primer instante una colaboración activa a favor de los jefes insurgentes comarcanos, entre sus actividades estaba reunir dinero, medicinas, armas, parque, vendas, pólvora, recibía noticias, enviaba correos y desempeñaba las tareas del más estricto espionaje ${ }^{6}$.

4 Salvo rarísimas excepciones, el mestizaje en México se encontró constituido por uniones de varones españoles con mujeres indígenas. La unión de estas mujeres con hombres españoles fue una transculturación profundamente dramática. La mujer se incorporaba brusca y violentamente a una cultura para la que no se encontraba preparada; su unión la llevaba a cabo traicionando su cultura original. Por tanto, el nacimiento de su hijo era la expresión de su alejamiento de un mundo, pero no la puerta abierta a otro distinto. El mestizo, fruto de la indígena con el español, buscaba inútilmente la aceptación del padre. La figura de la hispanidad quedó inevitablemente asociada con lo masculino. La mujer simbolizaba toda sociedad indígena reprimida, sumisa y vencida. LAVALLE URBINA, María, «Historia de la Mujer en México», México, 1988.

5 Doña Leona, en lucha con el cerrado criterio de su tío y albacea, esperaba la oportunidad para reunirse con el hombre de sus sueños; se carteaba con él, daba aviso de contingencias a los jefes insurgentes que se acercaban a México y se valía del arriero Mariano Salazar para enviar armas, pertrechos, víveres y ropa a los de Zitácuaro... Doña Leona logró recuperar la mayor parte de sus bienes; compartió con su esposo los triunfos y las nuevas vicisitudes; fue anti-iturbidista en razón de sus sentimientos democráticos y anti-bustamantista, para execrar el asesinato del general Vicente Guerrero. COVARRUBIAS, Ricardo, «Mujeres de México», Gobierno del Estado de Nuevo León, Dirección de Promoción Editorial, México. 1981, pp.81-82.

6 Todos los jefes insurgentes sabían que en casa de doña Gertrudis tenían abrigo, elementos, ayuda material y moral y pronto fue amonestada la infatigable mujer 
Doña Josefa Ortiz de Domínguez, por su parte, es el enlace entre los insurgentes. Induce a su esposo, el corregidor Miguel Domínguez, a participar en la conjura, además, envía un mensaje que sería clave a los insurgentes Hidalgo, Allende y Aldama, sobre que su esposo había sido obligado a catear la casa en donde se encontraban las armas de los sublevados ${ }^{7}$, entre otras muchas acciones; por lo que posteriormente fue encarcelada. Años después, el Congreso del Estado de Querétaro la declaró Benemérita.

\section{México Independiente: Siglo XIX}

Ya en el México independiente, en pleno siglo XIX, como antecedente de los movimientos feministas en México, cabe recordar la Carta de las Mujeres Zacatecanas, que desde 1824 solicitaban ser reconocidas como ciudadanas. Esta lucha, que se inicia propiamente en ese año, tardaría más de un siglo en lograr su triunfo.

Las ideas manejadas por Platón en torno a lo que debe ser el Estado estuvieron presentes en los preceptos de los liberales del siglo XIX mexicano, con don Benito Juárez, Sebastián Lerdo de Tejada y Vicente Riva Palacio, quienes consideraron la necesidad de crear instituciones para la educación de las mujeres. Juárez dio argumentos a favor de la secularización de la enseñanza y de los establecimientos educativos que habían estado en manos de la Iglesia desde la conquista, sostenía en su Programa de Gobierno de 20 de enero de 1861 que "secularizando los establecimientos de utilidad pública, se atenderá también a la educación de las mujeres, dándoles la importancia que merecen por la influencia que ejercen en la sociedad» ${ }^{8}$.

que cesara en sus propósitos, amenazándola en caso contrario a ser aprehendida y procesada... la rotunda negativa de doña Gertrudis para denunciar a sus colaboradores, hizo que el jefe de la guarnición la condenara a muerte. Ibídem, pp. 27-28.

7 Era el 14 de septiembre cuando Doña Josefa, encerrada con llave por su esposo el Corregidor, llamó desde su recámara en forma convenida al alcaide Ignacio Pérez; éste advirtió la urgencia de la llamada puesto que la Corregidora golpeó con el tacón de su calzado... le ordenó doña Josefa que sin pérdida de momento ensillara un caballo y se encaminara a San Miguel el Grande a enterar al capitán Allende de lo que pasaba en Querétaro. Pérez obedeció y el aviso de la Corregidora determinó la proclamación de la Independencia. Ibídem,p. 67.

8 Programa de Gobierno del Presidente Benito Juárez, 20 de Enero de 1861, en México a través del informe presidencial. La educación Pública, México, Ed. Secretaría de Educación Pública y Secretaría Presidencial, 1976, citado por LAVALLE URBINA, María, op. cit., 1988, p. 26. 
Así mismo, en 1883, comenzó a publicarse en México El Álbum de la Mujer, dirigido por Concepción de Flaquer y el Correo de las Señoras.

Posteriormente, en 1884, Luz Bonequi conquistó el primer título de telegrafista para la mujer y trabaja en la oficina Central de Telégrafos; en 1886, Margarita Chorné se convirtió en la primera profesionista con título, el de dentista, expedido por la Junta Directiva de Instrucción Pública del Distrito Federal. Al año siguiente, Matilde Montoya se convirtió en la primera médica titulada de México; le siguieron Columba Rivera, Guadalupe Sánchez y Soledad Regules.

Muy importante resultó a finales del Siglo XIX la figura de la periodista y escritora guerrerense Laureana Wright González, quien luchó por el sufragio y la igualdad de las mujeres. Autora que causó gran polémica en 1888 con La emancipación de la mujer por medio del estudio, fundó la primera revista feminista de México, Violetas del Anáhuac ${ }^{9}$, así como el periódico Mujeres del Anáhuac, desde cuyas páginas luchó por el sufragio de las mujeres y la igualdad de oportunidades en todos los terrenos. En sus artículos destacaba los avances que las sufragistas habían logrado en Estados Unidos y clamaba por la igualdad de derechos de los dos sexos como la verdadera regeneración de la humanidad ${ }^{10}$.

Aunque años atrás, en 1875, ya se habían realizado intentos por el Presidente Lerdo de Tejada de que se convirtiera la Escuela Secundaria de Niñas en la Escuela Normal para Señoritas, es hasta 1888 cuando la Escuela Secundaria se convierte en la Normal de Profesoras, con aprobación del Congreso. Y al año siguiente, en 1889, se titularon las primeras abogadas, María Asunción Sandoval de Zarco y Josefina B. de Arce. Unos años más tarde, en 1896, Guadalupe F. de Gómez inició la publicación del periódico Las Señoras.

A finales del siglo XIX y principios del XX, las mujeres también empezaron a organizarse en clubes políticos. Así, las ideas libertarias contra la dictadura de Porfirio Díaz llevaron aparejada la organización de las mujeres. Basta recordar el trabajo del Club Liberal Ponciano Arriaga en San Luis Potosí en 1900, en oposición al régimen porfirista, con ramales en todo el país. Destacaron, en Zitácuaro, Michoacán, Aurora y Elvira Colín; en Veracruz, Josefa Arjona de Pinelo y Donaciana Salas; en Coatzacoalcos, Veracruz, Josefa Tolentino y Rafaela Alor; en Chihuahua, Silvana Rembao de Trejo; en la ciudad de México, Concepción Valdés, Modesta Abascal, Otilia y Eulalia

9 Ibídem, p. 40.

10 Idem.

(C) UNED. Revista de Derecho UNED, núm. 13, 2013 
Martínez Núñez, Asimismo, se crearon asociaciones femeniles de apoyo al Club Liberal Ponciano Arriaga; en Veracruz, el Club Liberal de Señoras y Señoritas discípulas de Juárez, y en Matehuala, San Luis Potosí, el Club Liberal de Señoras y Señoritas, de Antiania Nava.

\section{Inicios del Siglo $\mathrm{XX}$}

No cabe duda que la igualdad es un camino largo, basta con recordar que en México hace apenas 60 años se les otorgó a las mujeres el derecho al voto, y fue hasta 1953 que se le reconoció la ciudadanía plena con el derecho a votar y ser votada ${ }^{11}$. En 1901 aparece en Morelia, Michoacán, el periódico mensual La Mujer Mexicana.

En Guanajuato, Juana Belén Gutiérrez de Mendoza funda Vésper, diario para la defensa de los mineros con posición anticlericalista y antiporfirista, por lo que las autoridades confiscan la imprenta. El romanticismo del siglo XIX se extiende al siglo XX. La imagen de las mujeres o es la tradicional o sugiere rebelión. Mujeres como Juana Belén Gutiérrez, Elisa Acuña y María del Refugio Vélez, en 1903 se integraron a la mesa directiva del Club Liberal Ponciano Arriaga de la ciudad de México.

En 1904 Laura N. Torres funda el grupo Admiradoras de Juárez, cuya finalidad es promover la emancipación de las mujeres. María Sandoval de Zarco y Laura Méndez de Cuenca fundan la Sociedad Protectora de la Mujer; La Mujer Mexicana, editado por Dolores Correa Zapata, es su órgano de difusión, donde pugnan por la superación y los derechos de las mujeres y los desvalidos.

Para 1906 se encabezaron varias agrupaciones entre ellas las Admiradoras de Juárez, con las profesoras Eulalia Guzmán, Hermila Galindo y Luz Vera, cuyo objetivo es la obtención del sufragio. Por su parte, Concepción Gómez Pezuela, Manuela M. de Oviedo y Emmy Ibañez Navarro fundan la Sociedad Internacional Femenina Cosmos y su publicación La Abeja. En Chihuahua, la señora Flores de Andrade establece el Club Hijas de Cuauhtémoc, con la finalidad de apoyar al Partido Liberal Mexicano ${ }^{12}$.

11 ... increíblemente, la mujer estaba relegada a un segundo plano, como los niños que deben conducirse de la mano de un adulto porque no tiene criterio para tomar sus propias decisiones. LÓPEZ SÁNCHEZ, Laura Paula, «El Valor de la Mujer», H. Congreso del Estado de Nuevo León, México, 2007, p. 77.

12 Este Programa fue suscrito el $1^{\circ}$ de Julio de 1906, por los dirigentes del Partido Liberal, encabezados por Ricardo Flores Magón, destacándose que todos ellos apoyaban al Partido. Por su parte, en su programa, "el Partido Liberal incluía el 
En 1907, Lucrecia O. Toríz, la «Virgen roja» de los trabajadores mexicanos, se enfrenta a los soldados durante la huelga general de la industria textil de Puebla junto a otras mujeres, levantan las armas. Mientras que en Atizapán de Zaragoza, Estado de México, se funda la agrupación de obreras textiles Hijas de Anáhuac, dirigida por las hermanas María del Carmen y Catalina Frías, Justa Vega y Elígia Pérez, para apoyar el movimiento magonista y el mejoramiento de las condiciones de trabajo de la mujer. En El Paso, Texas, Isidra T. Cárdenas publica el semanario La Voz de la Mujer, y participa en la campaña libertadora de los Flores Magón.

En 1909 en todo el país, surgen asociaciones antirreeleccionistas y clubes femeniles; en Chihuahua, el Sara Pérez de Madero, organizado por Dolores Romero de Revilla. En la Cd. de México, la Liga Femenil de Propaganda Política, dirigida por María Luisa Urbina, Joaquina Negrete y Adela Treviño. En Puebla, el Club Femenil Antirreeleccionista Josefa Ortiz de Domínguez, integrado por mayoría de trabajadoras cigarreras. También en Puebla, la Junta Revolucionaria de Mujeres, encabezada por las maestras normalistas Carmen Serdán, Paulina Maraver, las hermanas Narváez e Ignacia Vázquez. En Guadalajara, el Club Antirreeleccionista Femenil Leona Vicario, en el que participa María Enríquez Hermosillo.

Mientras que en Estados Unidos, las magonistas Andrea Villarreal, la «Juana de Arco mexicana», y Mary Harris Jones «Mother Jones», luchan por la libertad de los miembros del Partido Liberal Mexicano. Bajo la dirección de Villarreal, en San Antonio, Texas, se publica el periódico Mujer Moderna, que apoya la causa magonista y lucha por la reivindicación de la mujer. Juana Belén Gutiérrez y Dolores Jiménez y Muro fundan el Club Político Femenil Amigas del Pueblo y se unen a Francisco I. Madero.

En 1910 en San Pedro, Coahuila, tomando en cuenta todas estas agrupaciones que se habían extendido por el país, Madero incluye en el discurso el tema valor cívico de la mujer, y las diversas asociaciones feministas se unen a él, entre ellas el recién creado Club Femenil Antirreeleccionista "Las Hijas de Cuauhtémoc», presidido por Dolores Jiménez y Muro, Hijas de Juárez y Josefa Ortiz de Domínguez inician actividades antirreeleccionistas.

tema de la mujer y hacía ver que el trabajo que desempeñaba en el hogar debía ser reconocido como tal y advertía que el trabajo doméstico de las mujeres realizado a domicilio debía ser remunerado adecuadamente»; citado por LAVALLE URBINA, María, op. cit., p. 44. 
En Puebla surge el Club Carmen Serdán. Romaina Salazar de Martínez organiza el Grupo Magisterial Revolucionario, Elisa Acuña Rossetti dice a Madero que fundará el periódico «La Guillotina» ${ }^{13}$, con la finalidad de activar la práctica revolucionaria, pues los discursos no son suficientes para evitar la reelección, que poco tiempo después, en la glorieta de Colón de la Ciudad de México, protesta por el fraude en las elecciones y reclama la participación política de las mujeres mexicanas. Se reorganiza la Universidad Nacional, con 14 mujeres por cada cien graduados.

Debemos recordar, por otra parte, que en mayo de 1911 varios centenares de mujeres solicitaron al Presidente Interino Francisco León de la Barra su derecho a votar, con el argumento de que éste no estaba excluido en la Constitución de 1857, ya que la Carta magna no se refería al sexo de los votantes. Esto habla de que en nuestro país, a pesar del retraso con que las mujeres lograron conquistar sus derechos, hubo mujeres y hombres que tuvieron conciencia de la necesidad de cambiar los atavismos imperantes.

Con el mismo espíritu, en 1911, Dolores Jiménez y Muro funda Regeneración y Concordia, organización pro derechos de las mujeres. Dolores es hecha prisionera, luego que se descubre en Tacubaya un complot de maderistas encabezado por ella, Gildardo y Rodolfo Magaña y José Vasconcelos. Redacta el Plan Político Social, que circula de forma clandestina en diversas entidades. Más grupos feministas se unen a la causa revolucionaria. Las sufragistas mexicanas solicitan a Emilio Vázquez Gómez se les otorgue el voto.

En 1912 el Club Las Hijas de Cuauhtémoc solicita el apoyo para Madero. Y se crea la Casa del Obrero Mundial. Entre sus miembros fundadores destaca María Hernández de Zarco, quien trabajó como cajista en la imprenta de Adolfo Montes de Oca.

En 1913 se funda el Comité Femenil Pacificador integrado por Isabel Medina, María Hernández Hoyo y Concepción Durán y como consecuencia del asesinato de Madero y Pino Suárez, Ela Adela Elodia Arce Vda. de Arciniega reorganiza el Club Las Hijas de Cuauhtémoc, pero ahora con el nombre de Hijas de la Revolución. Crean diversas agrupaciones pero ahora luchan contra el gobierno huertista. Las Amigas del Pueblo publican un manifiesto en protesta por los sucesos de la Decena Trágica ${ }^{14}$.

13 VIDALES, Ismael, ELIZONDO, Daría, RODRÍGUEZ, Gerardo, «La Perspectiva de Género», Breve Estudio en Nuevo León, 1. ${ }^{a}$ ed., Ed. CECyTE, Nuevo León, México, 2007, p. 47.

14 VIDALES, Ismael, ELIZONDO, Daría, RODRÍGUEZ, Gerardo, op. cit., p. 48-49. 
También con la experiencia que tenía y ya pasados los años, Juana Belén Gutiérrez de Mendoza funda en 1914 el diario La Reforma, que reivindica a la raza indígena y con el mismo propósito pero en Cuernavaca, Morelos, Dolores Jiménez y Muro y Aurora M. Martínez publican el periódico antihuertista La Voz de Juárez, motivo por el que son perseguidas. Hermila Galindo, representante del Club Abraham González, pronuncia un discurso de bienvenida a Venustiano Carranza a su llegada a la capital, y se convierte en su secretaria. Consuelo Uranga representa a México ante el Congreso Feminista de París, que se pronuncia en contra de la guerra. Se legaliza el divorcio, el derecho a la pensión alimenticia y al manejo y propiedad de bienes. Emiliano Zapata nombra a Aurora Martínez de Hernández agente del Ejército Libertador del Sur.

Mientras que Salvador Alvarado asume, en 1915, a la gubernatura de Yucatán, expide un código sanitario para el régimen de la prostitución; es importante destacar aquí que al hablar de feminismo en México en el Siglo XX es preciso hacer un reconocimiento muy especial a este personaje en la historia; es decir, a Salvador Alvarado, quien bajo el influjo del movimiento revolucionario buscaba el cambio y tuvo conciencia de la necesidad de que en una sociedad todos sus miembros participaran en la toma de decisiones y en su desarrollo. Sabía que la mejor inversión que puede hacer el Estado es la educación de las mujeres para lograr el desarrollo integral de la población, fue por ello que impulsó importantes cambios a favor de las mujeres.

Salvador Alvarado, congruente con las medidas administrativas en pro de las mujeres que había dictado en su Estado, organizó el Primer Congreso Feminista de Yucatán, del 13 al 16 de enero de 1916, en cuya convocatoria señalaba los propósitos que lo llevaban a celebrar ese histórico encuentro. ${ }^{15} \mathrm{Si}$ la convocatoria resultó un documento acertado, con mayor razón al momento de celebrarse el mismo; era de tomarse en cuenta el Informe del Congreso, que quedaría como sentencia para la posteridad aún y cuando todavía faltaba un buen tramo por recorrer. ${ }^{16}$

15 «Es un error educar a la mujer para una sociedad que ya no existe, habituándola a que, como en la antigüedad, permanezca recluida en el hogar, el cual sólo abandona para asistir a los saraos y fiestas religiosas, y que no se le reivindica colocando sobre su tumba el epitafio romano: "cuidó su casa y supo hilar la lana", pues la vida activa de la evolución exige su concurso en una mayoría de actividades humanas». El Primer Congreso Feminista de Yucatán. Talleres Tipográficos del Ateneo Peninsular, 1916, p. 31 citado por CARPIZO, Jorge, «Derecho a la Información y Derechos Humanos», Ed. UNAM, México, 2000, p. 68.

16 «Puede la mujer del provenir desempeñar cualquier cargo público que no exija vigorosa constitución física, pues no habiendo diferencia alguna entre su estado 
A dicho Congreso asistieron 617 delegadas, entre las que destacó la participación de Hermila Galindo de Topete, quien en su ponencia «La mujer del porvenir» planteaba la igualdad intelectual entre la mujer y el hombre, al tiempo que demandaba la máxima libertad sexual para ambos sexos y el derecho al voto para la población femenina. También en 1916, la entonces secretaria de Venustiano Carranza, junto con Edelmira Trejo de Mellón, envió al Congreso una propuesta para que se otorgara el voto a la mujer, la cual fue rechazada.

En el Congreso había resabios de la sociedad porfirista que no veía con muy buenos ojos, por ejemplo, la conquista femenina de acceder a la educación superior, considerando que quienes seguían una carrera profesional tendían a masculinizarse o, aún peor, veían un verdadero peligro en las mujeres, ya que sería una incursión en un campo que hasta ese entonces se encontraba vedado para ellas, motivo por el cual se pretendía la limitación a su desarrollo ${ }^{17}$.

Otra limitante se imponía cuando existía oposición de otorgarles a las mujeres el derecho al sufragio, y los argumentos eran tan variados como insostenibles, por ejemplo, algunos consideraban que darles el voto a las mujeres era darle doble voto al varón, porque ellas ejercerían su derecho según lo que su pareja les indicara. Otros señalaban que equivaldría a darle voto al clero, ya que las mujeres eran totalmente dominadas por la Iglesia. Finalmente, otros sostenían el sofisma de que no había necesidad de hacer reformas constitucionales, porque cuando se hablaba del voto de los mexicanos, esto incluía también a las mujeres, aunque tal derecho nunca había sido puesto en práctica.

En este mismo año surgen en México diversos movimientos, como son la Confederación de Sindicatos del Distrito Federal, encabezada por los electricistas; se declara la huelga general, y el presidente Carranza ordena aprehender a los dirigentes, entre ellos Esther Torres y Ángela Inclán, quienes son absueltas de la pena capital luego de que los huelguistas fueron juzgados; sin embargo, reclaman igualdad en las responsabilidades, argumentando que tienen la misma representación, por lo que Carranza ordena se castigue por igual a hombres y mujeres. Inicia sus trabajos el Congreso Constituyente en Querétaro y Hermila Galindo envía al Constituyente una

intelectual y el del hombre, es tan capaz como éste de ser elemento dirigente de la sociedad». Ibídem, p. 70.

17 «Somos partidarios de la instrucción de las mujeres, pero no quisiéramos la multiplicación de las cerebrales, PALAVICINI, Félix F., "Problemas de la Educación», México, Ed. F. Sampere y Compañía, 1910, p. 67, citado por CARPIZO, Jorge, op. cit., p. 70. 
propuesta para que se otorgue el voto a las mujeres, que es apoyada por el representante de Michoacán, Salvador González Torres. Por el contrario, Inés Malváez presenta otra propuesta, pero en contra de que se otorgue el sufragio a las mujeres, Galindo encabeza un nutrido grupo de mujeres que se manifiesta por el voto a las puertas del Teatro Iturbide de Querétaro, donde se celebraba el Congreso Constituyente.

De diciembre de 1916 a enero de 1917, durante los debates del Congreso Constituyente, Félix F. Palavicini pide que se le explique por qué no se toman en consideración las iniciativas relativas al voto femenino. Pero el 26 de enero de 1917, en la 63. ${ }^{\text {a }}$ Sesión Ordinaria del Congreso Constituyente, sin discusión, por 166 votos contra 2, fue aprobado el artículo 34. Su texto quedó así:

"Artículo 34. Son ciudadanos de la República todos los que, teniendo la calidad de mexicanos, reúnan, además, los siguientes requisitos:

I. Haber cumplido diez y ocho años siendo casados, o veintiuno si no lo son;

II. Tener un modo honesto de vivir ${ }^{18}$ 》.

Con la aprobación del artículo 34, el 5 de febrero de 1917, se promulga la Constitución Política de los Estados Unidos Mexicanos, la que hoy en día se encuentra en vigencia en el país, con todas las modificaciones, cambios de fondo y de forma, que la hace parecer un documento diverso.

\section{Etapa posterior a la Constitución de 1917}

Bajo el amparo de la nueva Constitución, en ese año de 1917, Hermila Galindo lanzó su candidatura para diputada por el V Distrito electoral de la Ciudad de México, pero no accedió a la legislatura al no ser favorecida por el voto, toda vez que no contó con el apoyo de las mujeres, mismas que no podían emitir su sufragio. A este respecto, un par de años más tarde, Salvador Alvarado publicó su obra "La Reconstrucción de México», en la que dio a conocer su pensamiento "feminista» ${ }^{19}$, fue precisamente en este contexto, que años más tarde se le concedió a las mujeres el voto provincial y el

18 Diario de Debates del Congreso Constituyente 1917, 63. ${ }^{a}$ Sesión Ordinaria. Tomo III, Ed. UNAM, 1976.

19 «¿Por qué negarle el derecho al sufragio, en asuntos municipales, por ejemplo, si la ciudad no es sino un conjunto de hogares y la mujer el sostén directo, el hogar donde se desarrolla la vida familiar?, LAVALLE URBINA, María, op. cit., p. 52. 
general, cuando su educación cívica le permitiera desempeñar esas funciones electorales a conciencia, y los puestos de elección popular le pudiesen ser confiados. En resumidas cuentas, no se pretendía el sufragio universal.

A lo largo del mes de septiembre de 1921 se festejó el Primer Centenario de la Consumación de la Independencia de México, el sentir libertario se extendía pero pocas eran las mujeres que se aceptaban como «luchadoras feministas», y la sociedad continuaba legitimando la discriminación mientras un espíritu nacionalista invadía el país. Por lo tanto, Felipe Carrillo Puerto, gobernador de Yucatán de 1922 a 1924, envió a la Legislatura del estado una iniciativa que concedía el voto a las mujeres; en consecuencia, este año, la profesora Rosa Torres G., obtuvo el primer cargo de elección popular en la República Mexicana, al ser electa primera regidora en el Ayuntamiento de Mérida, ocupando así la Regiduría de Prensa y Beneficencia, cargo que cumplió de principio a fin.

El Partido Socialista del Sureste, encabezado por Carrillo Puerto, lanzó la candidatura de tres diputadas propietarias: Elvia Carrillo Puerto, Beatriz Peniche y Raquel Dzib Cicero, lamentablemente ninguna de las diputadas pudo ejercer plenamente sus funciones. Al caer el gobierno de Carrillo Puerto, estas elecciones, en las que salieron triunfadoras, fueron anuladas. Pero Elvia Carrillo Puerto, hermana del gobernador, el 18 de noviembre de 1923, se convirtió en la primera mexicana electa diputada al Congreso de Yucatán, por el V Distrito. Después de desempeñar el cargo durante dos años, presentó su renuncia por diversas presiones, entre ellas: amenazas de muerte, por lo que, posteriormente, en 1925, cambió su residencia de Yucatán a San Luis Potosí, y se presentó a las elecciones legislativas de este estado. No obstante que obtuvo la mayoría de votos, el Colegio Electoral no le reconoció el triunfo, lo que ocasionó que en diversas partes de la República hubieran acciones de apoyo, semejantes a la anteriormente realizada, del 20 al 30 de mayo de 1923, en la Ciudad de México, lugar en el que se celebró el Primer Congreso Nacional Feminista, convocado por la sección mexicana de la Liga Panamericana de Mujeres.

Cabe mencionar que entre las resoluciones del Primer Congreso Nacional Feminista están: solicitar la igualdad civil para que las mujeres puedan ser reelegibles en los cargos administrativos y promover el decreto de la igualdad política y la participación de agrupaciones sociales en la representación parlamentaria. 
Posteriormente a dicho Congreso, en San Luis Potosí, el 13 de junio de 1923, se expidió el decreto que concedió a las mujeres el derecho a votar y a ser electas en elecciones municipales, siempre y cuando supieran leer y no pertenecieran a ninguna asociación religiosa; sin embargo, tiempo más tarde estos derechos fueron derogados.

Es hasta 1929 cuando volvieron a surgir nuevas asociaciones femeninas, cuyo principal objetivo es obtener los derechos políticos plenos: el Partido Feminista Revolucionario y el Bloque Nacional de Mujeres Revolucionarias. En 1931, 1932 y 1934, se llevaron a cabo tres Congresos Nacionales de Obreras y Campesinas, de donde surgen diversas organizaciones femeninas, entre ellas, el Frente Único Pro Derechos de la Mujer.

Durante la campaña presidencial del General Lázaro Cárdenas, en 1934, se dio la mayor organización de mujeres hasta entonces, y se conformó el Frente de Mujeres Mexicanas. Por su parte, Carlos Riva Palacio, líder del Partido Nacional Revolucionario, convocó a la formación del sector femenino del partido, que hasta entonces se dividía en tres sectores, Obrero, Campesino y Militar. Las principales asociaciones de mujeres que se sumaron a las filas del PNR ${ }^{20}$ fueron: La Liga Orientadora de Acción Femenina (creada en 1927), dirigida por Elvia Carrillo Puerto; El Bloque Nacional de Mujeres Revolucionarias (creado en 1929), presidido por Florinda Lazos León; El Partido Feminista Revolucionario (creado en 1929), de donde saldría la primera dirigente del Sector Femenil del PNR, Edelmira Rojas Vda. de Escudero; y la Confederación Femenil Mexicana (creada en 1931), encabezada por María Ríos Cárdenas.

Después de muchos intentos, en 1935 se alcanzó el primer triunfo en cuestiones partidistas. Por primera vez, las mujeres participaron en las votaciones internas de un partido político; trabajadoras y campesinas de Veracruz votaron en las elecciones para designar los comités municipales del PNR y de las autoridades de los ayuntamientos. El Frente Único Pro Derechos de la Mujer, reunió a 800 agrupaciones femeninas de todo el país con cerca de 50 mil miembros de diversas posturas ideológicas que compartían un mismo objetivo: conquistar el derecho a votar y ser electas.

Este mismo año, el 1 de septiembre, en su primer informe de gobierno, el Presidente Lázaro Cárdenas señaló la necesidad de que el PNR buscara un mayor acercamiento con las masas, particular-

20 PNR, Partido Nacional Revolucionario, antecedente del actual, Partido Revolucionario Institucional, PRI. 
mente con los sectores juvenil y femenil. En consecuencia, el PNR transformó la sección femenina en una Oficina de Acción Femenina, dependiente del Comité Ejecutivo Nacional.

Mientras tanto, en Veracruz, el 30 de marzo de 1936, el PNR lanzó la candidatura para diputadas locales de dos mujeres: María Tinoco, como propietaria, y Enriqueta L. de Pulgarón, como suplente; a pesar del apoyo, el registro no fue aceptado por el Departamento Electoral Nacional, en vista de que la Ley Electoral no incluía ningún capítulo sobre las mujeres.

También se alcanzó el segundo triunfo en cuestiones partidistas: En la Ciudad de México, las activistas del PNR conquistan su derecho a participar en las elecciones internas de su partido para postular candidatos a gobernadores y senadores para el período 1936-1940. En esa tesitura, el 4 de abril de 1937, se alcanzó el tercer triunfo en cuestiones partidistas, cuando todas las mujeres miembros regulares de uniones campesinas, sindicatos, ligas agrarias y de las propias secciones femeninas votan en las elecciones sectoriales del PNR celebradas ese día en todo el país.

Sin embargo, conscientes de sus alcances, continúan luchando por la igualdad política plena, inconformándose por lo que la cúpula del partido les había «concedido». Por ello, el Frente Único Pro Derechos de la Mujer apoya las candidaturas de Soledad Orozco para diputada local por León, Guanajuato, y de Refugio García para diputada federal por Uruapan, Michoacán.

Pese al apoyo popular, el mismo 4 de abril la Convención del PNR, hasta esa fecha integrada sólo por varones, designó a otros candidatos. En junio de 1937, Soledad Orozco y Refugio García continúan sus campañas sin apoyo partidista, lo que las convierte en las primeras candidatas independientes. En junio solicitan a la Suprema Corte de Justicia su intervención para que se reconozca la supremacía de los artículos 34 y 35 de la Constitución, argumentan que éstos concedían la ciudadanía a todos los mexicanos por encima del artículo 37 de la Ley Electoral Federal, que concedía el sufragio exclusivamente a los hombres. Pero prevaleció la idea de que las mujeres no podían votar ni ser votadas si antes no se reformaba la Constitución.

El 26 de agosto de 1937 el Presidente Cárdenas anuncia en Veracruz, en un mitin de la Confederación Femenina Mexicana, una declaración a la prensa que pasaría a la historia como la primera 
manifestación sobre el derecho de las mujeres, proveniente del Presidente de la República ${ }^{21}$.

En su tercer informe de gobierno, el $1^{\circ}$ de septiembre de 1937, el Presidente Cárdenas señala la necesidad de rehabilitar los derechos políticos de las mujeres. Y el 19 de noviembre, envía a la Cámara de Senadores la iniciativa de reformas al artículo 34 constitucional, como primer paso para que las mujeres obtengan la ciudadanía, acción que recibieran con beneplácito las mujeres organizadas dentro del PNR en el Distrito Federal.

Así mismo, el 27 de septiembre de 1937 la historia registra una carta de Evangelina A. de Vaughan, presidenta del Consejo Internacional de la Unión de Mujeres Americanas, dirigida al Presidente Lázaro Cárdenas, en la que, en nombre de 200 mil mujeres, lo felicitan por la forma magistral con que ha defendido los derechos de las mujeres mexicanas en su mensaje al Honorable Congreso.

El 23 de noviembre de 1937, la iniciativa de reformas del Presidente Cárdenas es recibida por las Cámaras. Y el 10 de diciembre de 1937 se emite tan sólo un dictamen sobre la iniciativa de reformas al artículo 34 constitucional. A principios de este año el Frente Único Pro Derechos de la Mujer decide incorporarse al PNR, considerando que bien valía perder la autonomía en aras de hacer ver a los opositores del movimiento femenino que no eran «ni mochas ni comunistas y que su lealtad estaba con la Revolución».

En Chilpancingo, Guerrero, Aurora Meza Andraca se convierte en la primera presidenta municipal. El $10^{\circ}$ de abril se crea el Partido de la Revolución Mexicana. En su Pacto Constitutivo se compromete a que las mujeres tengan "completa igualdad con los elementos masculinos». Asimismo, la Declaración de Principios demanda la modificación inmediata de «la situación de inferioridad en que la mujer ha vivido respecto al hombre». El 6 de julio de 1938 el Presidente Cárdenas envía el proyecto de reformas al artículo 34 constitucional a la Cámara de Diputados, la que, a su vez, lo envía a las Legislaturas locales, algunas de las cuales emiten su voto.

Por otra parte, la situación política de México hizo suponer al PRM que el candidato de oposición Juan Andreu Almazán, del Partido Revolucionario de Unificación Nacional, se beneficiaría del voto

21 «el gobierno no se detendrá únicamente a tratar cuestiones que interesen o más convengan a los hombres, sino que colocará a las mujeres en el mismo plano que a los hombres, y para tal efecto, presentaré a las cámaras las reformas necesarias para que las mujeres queden definitivamente incorporadas a la función social y política», VIDALES, Ismael, ELIZONDO, Daría, RODRÍGUEZ, Gerardo, op. cit., p. 51. 
femenino en las elecciones de 1940, otro posible motivo por el que la iniciativa cardenista no prosperó. Sin embargo, esto no detuvo al movimiento feminista y dirigidas por Refugio Rangel Olmedo, Lucina Villarreal y Aurora Fernández, las mujeres se organizan en ligas femeninas. La Unión de Mujeres Americanas realiza manifestaciones para presionar a la Cámara de Diputados. Solicitan en particular la modificación del artículo 37 de la Ley Electoral de poderes federales, que señalaba sólo como ciudadanos a los varones.

El 1. ${ }^{\circ}$ de septiembre de 1939, el Presidente Cárdenas reitera su iniciativa de reformas y explica que "por falta de declaratoria» no se ha llevado a cabo la reforma constitucional. En noviembre de 1939, en la Primera Asamblea Nacional del PRM se formula el Segundo Plan Sexenal y las mujeres consiguen que se incluya una intención de enmienda a la Constitución y a la Ley Electoral para que las mujeres obtengan el derecho al voto. Durante los años de 1940 y 1941, en los inicios del gobierno de Manuel Ávila Camacho, las secretarías femeniles de las organizaciones internas del PRM constituyen la Alianza Nacional Femenina.

El 18 de enero de 1946, el Partido de la Revolución Mexicana cambia su nombre por el de Partido Revolucionario Institucional. En sus documentos constitutivos se establece que se fortalecerá la participación femenina en general, además de que se eleva el número de delegadas en el Consejo Nacional.

El día 4 de diciembre de 1946, tres días después de haber iniciado su periodo presidencial, Miguel Alemán envía a la Cámara de Senadores la iniciativa de adición al artículo 115 de la Constitución, por la que otorga el derecho a las mujeres de votar y ser votadas en elecciones municipales. Esta iniciativa fue aprobada, después de interesantes debates, el martes 31 de diciembre de 1946, en Sesión Ordinaria de la Cámara de Senadores. Se publicó en el Diario Oficial de la Federación del 12 de febrero de 1947, fecha en que entró en vigencia $^{22}$.

En el año de 1947, a raíz de la reforma del artículo 115 de la Constitución, las mujeres comenzaron a ocupar cargos de elección en los Ayuntamientos: En Aguascalientes, María del Carmen Martín del Campo se convierte en la primera Presidenta Municipal de esta ciudad. Ya antes algunas mujeres habían ocupado presidencias mu-

22 Artículo 115 de la Constitución Política de los Estados Unidos Mexicanos: «... en las elecciones municipales participarán las mujeres en igualdad de condición que los varones, con derecho a votar y ser votadas. Diario Oficial de la Federación, 31 de diciembre de 1946. 
nicipales, recordamos que la primera fue Aurora Meza Andraca, y poco después Virginia Soto, en Dolores Hidalgo, Guanajuato. Mientras que en el Distrito Federal, en 1947, Aurora Fernández es nombrada delegada de Milpa Alta, y Guadalupe Ramírez de Xochimilco.

El 6 de abril de 1952 se lleva a cabo en el Parque 18 de Marzo de la Ciudad de México, la Asamblea Nacional Femenil, en la que participan representantes de los tres sectores del Partido Revolucionario Institucional, de todos los Estados de la República, a favor de la candidatura de Adolfo Ruiz Cortines por la Presidencia de la República, en donde se calcula una asistencia de 20 mil mujeres. Fue ante ellas que el Presidente Ruiz Cortines promete iniciar las reformas constitucionales para reconocer a las mujeres la igualdad política ${ }^{23}$.

El 1. ${ }^{\circ}$ de diciembre de ese mismo año, en su discurso de toma de posesión como Presidente de México, Adolfo Ruiz Cortines anuncia que enviará a las Cámaras una iniciativa para reformar el artículo 34 constitucional y demás relativos al voto femenino, con la finalidad de incorporar de manera plena y definitiva a las mujeres en la vida política nacional y al día siguiente envía al $\mathrm{H}$. Congreso su iniciativa de reformas a los artículos 34 y 115 de la Constitución de 1917. La iniciativa, además de los considerandos, señala:

"Artículo 1. ${ }^{\circ}$ Se reforma el artículo 34 de la Constitución Política de los Estados Unidos Mexicanos, para quedar en los siguientes términos:

Artículo 34. Son ciudadanos de la República, los varones y las mujeres que, teniendo la calidad de mexicanos, reúnan, además, los siguientes requisitos: I. Haber cumplido 18 años, siendo casados, ó 21 si no lo son, y II. Tener un modo honesto de vivir.»

En cuanto al artículo 115, fracción I, se propone la supresión de la frase relativa al voto de las mujeres en elecciones municipales, entre otros cambios.

En atención a la tendencia mundial, y toda vez que nuestro país participó de la inquietud mundial de reconocer los derechos de la mujer ${ }^{24}$, fue el motivo principal para que el 6 de octubre de 1953 en

23 «...si el voto nos favorece en los próximos comicios, nos proponemos iniciar ante las Cámaras las reformas legales necesarias para que la mujer disfrute los mismos derechos políticos del hombre». Y las mujeres gritaban: ¡Repítalo, Don Adolfo, repítalo! Se estableció un compromiso. Crónica de ANDRADE DE DEL ROSAL, Martha, citada en «La Lucha de las Mujeres», p. 77, Revista Géneros, p. 49.

24 ...y el 20 de diciembre de 1952 se lleva a cabo la Convención sobre los Derechos Políticos de la Mujer, con el deseo de poner en práctica el principio de igualdad de derechos de hombres y mujeres enunciado en la Carta de las Naciones Unidas. En ella se reconoce que toda persona tiene derecho a participar en el gobierno de su país, directamente o por conducto de sus representantes, con ello las mujeres en todo 
Sesión Ordinaria de la Cámara de Diputados, se declararan reformados los artículos 34 y 115, fracción I, de la Constitución Política de los Estados Unidos Mexicanos. La aprobación se dio por unanimidad de 98 votos. Y el13 de octubre de 1953 el Presidente Adolfo Ruiz Cortines expide la reforma de los artículos 34 y 115, fracción I, constitucionales. Por el primero, se otorga plenitud de derechos ciudadanos a las mujeres mexicanas y, en consecuencia, la adición al artículo 115, fracción I, relativa al voto de las mujeres en elecciones municipales y aprobada el 31 de diciembre de 1946, desaparece del texto constitucional. Las reformas fueron publicadas en el Diario Oficial de la Federación del 17 de octubre de 1953.

México continuaba poniendo sus ojos en los documentos internacionales, para su internación en el país, sobre todo, aquellos tendientes a reconocer los derechos de las mujeres ${ }^{25}$, y con base en este antecedente Aurora Jiménez de Palacios, el 7 de septiembre de 1954, se convierte en la primera diputada federal, por el 1er. Distrito del estado de Baja California, como resultado de las elecciones extraordinarias verificadas el 4 de julio de 1954 en esa entidad, rindiendo protesta ante la XLII Legislatura (1952-1955) el 7 de septiembre de 1954.

El día 3 de julio de 1955 las mujeres acuden a las urnas a emitir su voto para elegir diputados federales para la XLIII Legislatura $(1955-1958)^{26}$. La primera en depositar su voto fue Doña María Izaguirre de Ruiz Cortines. Resultaron electas: Remedios Albertina Ezeta, por el Estado de México; Margarita García Flores, por Nuevo León; Guadalupe Ursúa Flores, por Jalisco, y Marcelina Galindo Arce, por Chiapas.

Durante los años de 1958-1961, Macrina Rabadán se convierte en la primera diputada propietaria de la oposición, por el Partido Popular Socialista, durante la XLIV Legislatura. Entre los años 1964-1967 y 1967-1970, Alicia Arrellano Tapia y María Lavalle Urbina se convierten en las primeras dos senadoras de la República por el partido

lugar y momento podrán votar y ser elegibles para cualquier cargo público. MARTÍNEZ GARZA, Minerva E., "La Declaración Universal de los Derechos Humanos», a sesenta años de distancia, Ed. UANL, México, 2008, p. 10.

25 El 17 de diciembre de 1954 la Organización de las Naciones Unidas resuelve la aprobación de la Resolución 843 sobre las condiciones de la mujer en derecho privado; costumbres, antiguas leyes y prácticas que afectan la dignidad de la mujer como ser humano, buscando en todo tiempo la internación de documentos internacionales. Ibídem, p. 12.

26 En el ámbito de nuestro país, la mujer mexicana por primera vez participa activamente en las elecciones y ejerce su derecho al voto, el 3 de julio en las elecciones para el legislativo federal. Ibídem, p. 15. 
en el poder, representando a Sonora y Campeche, respectivamente, en las XLVI (1964-1967) y XLVII (1967-1970) Legislaturas. Lavalle Urbina llegó a ser presidenta del Senado ${ }^{27}$.

Con todos los cambios importantes que se vivieron en estos años, cabe mencionar que la lucha por la que se había pasado, se refleja cuando se promulga la reforma del segundo párrafo del artículo $4^{\circ}$ de la Constitución mexicana, mediante el cual, se eleva a rango constitucional la igualdad entre el varón y la mujer, así como la libertad sobre el número y espaciamiento de los hijos. Esta reforma se publica en el Diario Oficial de la Federación del 31 de diciembre de 1974 y su vigencia comienza el $1^{\circ}$ de enero de 1975 , quedando como sigue:

"Artículo 4. ${ }^{\circ}$ El varón y la mujer son iguales ante la ley. Ésta protegerá la organización y el desarrollo de la familia.

Toda persona tiene derecho a decidir de manera libre, responsable e informada sobre el número y el espaciamiento de sus hijos.»

Los pasos importantes encaminados a obtener la declaración de la igualdad que se habían plasmado en la historia, se ven consolidados en 1979 con Griselda Álvarez Ponce de León, quien se convirtió en la primera gobernadora de un estado de la República Mexicana, Colima. Ocupando el cargo hasta 1985, y en el periodo de 1988 a 1991, Ifigenia Martínez Hernández se convierte en la primera senadora de la oposición, en la LIV Legislatura (1988-1991). El 11 de septiembre de 1993, la Cámara de Diputados debate la fracción III del artículo 175 del Código Federal de Instituciones y Procedimientos Electorales. Este mismo día se aprueba la propuesta de varias diputadas que dice: "Los partidos políticos promoverán en los términos que determinen sus documentos internos, una mayor participación de las mujeres en la vida política del país, a través de su postulación a cargos de elección popular».

El 14 de noviembre de 1996, en debate de la Cámara de Diputados, las mujeres proponen y se aprueba una adición a la fracción XII Transitoria del artículo $1^{\circ}$ del Código Federal de Instituciones y Procedimientos Electorales, que señala: «Los partidos políticos nacionales considerarán en sus estatutos que las candidaturas a diputados y senadores no excedan el 70 por ciento para un mismo género. Así mismo, promoverán la mayor participación política de las mujeres ${ }^{28}$.

En el año 2002 se presenta nuevamente una reforma al Código Federal de Instituciones y Procedimientos Electorales, publicada

27 LAVALLE URBINA, María, op. cit., p. 69.

28 CARPIZO, Jorge, op. cit., p. 62. 
en el Diario Oficial de la Federación el 24 de junio de 2002, la cual permitiría avanzar hacia una democracia paritaria, la misma fue aprobada por la Cámara de Diputados por unanimidad y dispone que los partidos políticos promoverán y garantizarán la igualdad de oportunidades y la equidad entre mujeres y hombres en la vida política del país, a través de postulaciones a cargos de elección popular.

El Tribunal Electoral del Poder Judicial de la Federación indicó en el 2011 que del periodo comprendido entre 1996 hasta 2008 solamente el $1.9 \%$ de los juicios que se resolvieron tenían que ver con las cuotas de género, de lo que se desprende que las militantes no reclamaban sus derechos electorales y políticos. Sin embargo, en noviembre de 2011 el Tribunal Electoral ordenó, a través de una sentencia, que los partidos políticos debían postular un mínimo de $40 \%$ de mujeres candidatas, tanto titulares como suplentes, para diputaciones federales y senadurías, lo que representó una fuerte reprimenda para quienes se resistían a que las mujeres participaran como candidatas en los comicios.

Este recuento histórico sólo marca el inicio de la lucha, no así la igualdad plena y definitiva, es decir, se logra el reconocimiento en el cuerpo de la ley, en donde se ratifica que las mujeres y los hombres tienen iguales derechos y obligan a los estados pactantes a adoptar medidas enfocadas a promover los cambios que aseguren a las mujeres el acceso a la educación, a la salud, al desarrollo humano y a la paz. Razón la anterior que sería el motor para poner en marcha los documentos específicos, nacionales e internacionales a favor de los derechos de las mujeres.

\section{Siglo XXI}

A raíz del proceso de emancipación por el que las mujeres han transitado en nuestro país, con mayor énfasis durante los últimos sesenta años, los elementos materiales y simbólicos que ayudan a construir la identidad de género, se han reconfigurado de modo tal que algunas categorías como las laborales, sociales, familiares, políticas y de salud reproductiva, se han ampliado o acotado conforme a la nueva construcción de la identidad genérica ${ }^{29}$.

29 ÁLVAREZ, Melba, (2010), «La legislación con perspectiva de género en el Distrito Federal, ¿un cambio obligatorio en la cultura?» En El Cotidiano, vol. 25, núm. 160, marzo-abril, México, D.F., p. 24. 
Para lo anterior fue necesario que se reconociera, primeramente, que existía una condición de desigualdad y desventaja institucional y jurídica de las mujeres frente a los hombres, con el objeto de que, posteriormente, se elaboraran leyes que legitimaran las nuevas formas materiales y simbólicas en las que se está construyendo el nuevo orden social de las relaciones entre mujeres y hombres.

En consecuencia, en nuestro país se han promulgado una serie de leyes y desarrollado diversas acciones afirmativas que buscan cumplir con las obligaciones consistentes en prevenir, atender, sancionar y erradicar la violencia de género y otros flagelos que impiden el crecimiento y empoderamiento de las mujeres en el entorno doméstico y social, ya sea económico, laboral, político, etcétera; instrumentos y mecanismos que analizaremos en otra oportunidad.

En el Estado de Nuevo León, el devenir de las mujeres ha cursado en forma similar al entorno nacional; y entre las que han luchado por la reivindicación de los derechos de las mujeres, destacan María Elena Chapa Hernández como la primera mujer en alcanzar un escaño en el Senado de la República, apenas al inicio de la última década del siglo XX, en el periodo 1991-1997.

Asimismo, Blanca Judith Díaz Delgado, quien alcanzó otra Senaduría para Nuevo León en la LX Legislatura del Congreso Federal.

Extraordinariamente, Nuevo León cuenta en la actualidad con tres representantes mujeres en el Senado de la LXII Legislatura del Congreso de la Unión: María Cristina Díaz Salazar, Ivonne Álvarez García y Marcela Guerra Castillo.

Asimismo, por primera ocasión, en la capital del Estado, Monterrey, se cuenta con una mujer ocupando el cargo de Presidenta Municipal: Margarita Arellanes Cervantes, para el periodo 2012-2014.

Finalmente, quiero mencionar que en México existen miles de mujeres que día con día, anónimamente, hacen historia en la construcción de la tan anhelada igualdad de género.

Por otra parte, en 2010, la esperanza de vida en las mujeres era de 77.8 años, aproximadamente cinco años más que la estimada en los hombres.

Según datos censales de 2010, cuatro de cada diez personas (40.9\%) de 15 años o más se encuentran en rezago educativo, encontrando que esta condición se presenta en $64.4 \%$ de las mujeres de esa edad que viven en localidades menores de 2 mil 500 habitantes, 
porcentaje similar al de los hombres cuyo rezago es de $63.7 \%$ en el mismo rango de edad.

De acuerdo con datos del segundo trimestre de 2012 de la Encuesta Nacional de Ocupación y Empleo (ENOE), 43.5\% de las mujeres de 14 años y más forman parte de la población económicamente activa (PEA) en el país, de las cuales, 91.9\% combina sus actividades extra domésticas (trabajo y estudio) con quehaceres domésticos, situación que resulta contrastante con la de los varones, entre los cuales $54.5 \%$ cumple con esta condición.

De las mujeres ocupadas, $23.5 \%$ trabajan por cuenta propia, $2.5 \%$ son empleadoras y $9.2 \%$ no recibe remuneración por su trabajo. Dos de cada tres mujeres ocupadas $(64.8 \%)$ son subordinadas y remuneradas. El 44.7\% de estas mismas trabajadoras no cuentan con acceso a servicios de salud, más de la tercera parte (35.2\%) no cuenta con prestaciones y $44.1 \%$ labora sin tener un contrato escrito.

Asimismo, al 2012, las mujeres tienen 43 de las 128 curules en la Cámara de Senadores, lo que representa una de cada tres del total, y 184 escaños en la Cámara de Diputados (más de una tercera parte: 36.8 por ciento). La tendencia respecto a la integración de las mujeres en años anteriores, muestra que la proporción de diputadas y senadoras ha aumentado.

Ciertamente aún faltan asignaturas pendientes. La desigualdad, discriminación y violencia en contra de las mujeres sigue siendo palpable en la cotidianidad mexicana.

\section{BIBLIOGRAFÍA}

ALPONTE, Juan María: Mujeres, Crónica de una Rebelión Histórica, Editorial Aguilar, México, 2005.

ARIAS, Alán (coordinador): Multiculturalismo y Derechos Indígenas, El caso mexicano, 1. ${ }^{a}$ edición, Editorial Comisión Nacional de los Derechos Humanos, México, 2008.

BEAUVOIR Simone D': El Segundo Sexo, Editorial Siglo Veinte, México, 2005.

BEDOLLA, M.-BUSTOS, R.-DELGADO, B.-GARCIA B.: Estudios de género y feminismo II, Ediciones FONTAMARA-UNAM, México, 1993.

BEGNE, Patricia: Los derechos humanos y la mujer en México, Editorial Comisión Nacional de los Derechos Humanos, México, 1991. 
BENSADON, Ney: Los derechos de la mujer, 1. ${ }^{a}$ edición, Editorial Fondo de Cultura Económica, México, 1998.

BEUCHOT, Mauricio: Derechos Humanos, Historia y Filosofía, 1. ${ }^{a}$ edición, Editorial ITAM, México, 1999.

BURÍN, M.: Estudios sobre la subjetividad femenina, Mujeres y salud mental, Grupo Editorial Latinoamericano, Argentina, 1991.

CASSIN, ALCALA-ZAMORA, SPOLANSKY y varios: Veinte años de evolución de los Derechos Humanos, Editorial Instituto de Investigaciones Jurídicas de la UNAM, México, 1974.

CASTILLA ALFONSO, Laura: ;Mujer! Lucha por tu ser, 38. ${ }^{\text {a }}$ edición, Editorial Diana, México, 2009.

CENTRO DE DERECHOS HUMANOS: Recopilación de Instrumentos Internacionales, Volumen I, Primera parte de las Naciones Unidas, Nueva York, 1994.

CENTRO DE DERECHOS HUMANOS: Recopilación de Instrumentos Internacionales, Volumen I, Segunda parte de las Naciones Unidas, Nueva York, 1994.

COVARRUBIAS Ricardo: Mujeres de México, 1. ${ }^{\mathrm{a}}$ edición, Editorial Dirección de Promoción Editorial, Gobierno del Estado de Nuevo León, México, 1981.

CHARLOT, Bunch y otros: Los Derechos de la Mujer son los Derechos Humanos, Editorial Edamex, México, 2000.

DALTON, P.: Mujeres, diosas y musas: Tejedoras de la memoria, Editorial El Colegio de México, México, 1999.

FERNÁNDEZ, Ana María (compiladora): Las mujeres en la imaginación colectiva: Una historia de discriminación y resistencia, Buenos Aires, 1992.

FERNÁNDEZ, David: Los Derechos Humanos en México durante la transición sexenal, Editorial Universidad Iberoamericana, México, 1995.

GALEANA, Patricia (compiladora): La condición de la mujer indígena $y$ sus derechos fundamentales: seminario internacional, Coordinación de Humanidades, Coedición UNAM-Comisión Nacional de los Derechos Humanos, México, 1997.

GONZALBO AIZPURU, Pilar-ARES QUEIJA, Berta (coordinadoras): Las mujeres en la construcción de las sociedades iberoamericanas, Consejo Superior de Investigaciones Científicas, Escuela de Estu- 
dios Hispano-Americanos, Editorial Colegio de México, Centro de Estudios Históricos, México, 2004.

GONZALEZ, Ana y otros: Mujer y Educación, Editorial Grao, Barcelona, 2002.

GONZÁLEZ, Nazario: Los Derechos Humanos en la historia, Editorial Alfaomega, México, 2002.

GONZÁLEZ RAMOS, Juventino: Los Derechos Humanos en Nuevo León, 1. a edición, Monterrey, Nuevo León, México, 1991.

INSTITUTO NACIONAL DE LAS MUJERES: Perfil de mujeres $y$ hombres en México, 2. ${ }^{a}$ edición, Editorial Instituto Nacional de las Mujeres, México, 2004.

LAVALLE URBINA, María: Historia de la Mujer en México, Editorial Fondo de Cultura Económica, México, 1988.

LAVALLE URBINA, María: Para la Mujer, igual trato que al varón, Editorial Partido Revolucionario Institucional, 16. ${ }^{\text {a }}$ edición, México, 1975.

Le BARON, Natalie-MAIER, Elizabeth: De lo privado a lo público, treinta años de lucha ciudadana de las mujeres en América Latina, 2. ${ }^{a}$ edición, Editorial Siglo XXI editores, México, 2007.

LENIN, Vladimir Ilich: La emancipación de la mujer recopilación de artículos, Editorial Colombia Pepe, Medellín, 1957.

LOPEZ SANCHEZ, Laura: El Valor de la mujer, publicaciones Comisión de Equidad y Género, Editorial Congreso del Estado de Nuevo León, LXXI Legislatura, México, 2007.

LOZANO FUENTES José Manuel-LOPEZ REYES Amalia: Historia General de México, 2. ${ }^{a}$ edición, Editorial Publicaciones Cultural, México, 2006.

LOZANO FUENTES José Manuel-LOPEZ REYES Amalia: Historia Universal Contemporánea, 2. ${ }^{a}$ edición, Editorial Publicaciones Cultural, México, 2007.

MARTÍNEZ GARZA, Minerva: La Declaración Universal de los Derechos Humanos, a sesenta años de distancia, 1. ${ }^{a}$ edición, Editorial UANL, México, 2008.

MARTÍNEZ MORÁN, Narcisov Mujer y Derechos Humanos en Cuestiones Fundamentales de Género: Desarrollo, actuaciones y tratamientos, Obra colectiva, Ministerio de Trabajo y Asuntos 
Sociales-Fundación Luis Vives, Nueva edición, revisada, Madrid 2001. pgs. $65-145$

MARTINEZ QUINTERO, Violante: Mujer y Participación en las Organizaciones, Editorial Universidad Nacional de Educación a Distancia, Madrid, 2001.

MENAGE, Gilles. Historia de las Mujeres Filósofas, 1. a edición, Editorial Herder, España 2009.

NASH, Mary: Mujeres en el Mundo, Historia, retos y movimientos, Editorial Alianza, Madrid, 2004.

OLAMENDI TORRES, Patricia: La mujer en la legislación mexicana, Ediciones Senado de la República, LVII Legislatura, Comisión de Equidad y Género, México, 1998.

ONU MUJERESv El Progreso de las Mujeres en el mundo. En busca de la justicia, s.e., Nueva York, Estados Unidos, 2011.

PARTIDO REVOLUCIONARIO INSTITUCIONAL. COMITE EJECUTIVO NACIONAL: La reforma del partido en la voz de sus mujeres: Asamblea Nacional preparatoria del Consejo para la Integración de la mujer, Editorial PRI-Secretaría de Información y Propaganda, México, 1999.

PECES-BARBA MARTÍNEZ, Gregorio: Reflexiones sobre la evolución histórica y el concepto de Dignidad Humana, Instituto de Derechos Humanos «Bartolomé de las Casas» Universidad Carlos III de Madrid, Editorial Dykinson, S.L. Madrid España, 2005.

PECHADRE, Lyde e ROUDY, Yvette: El Éxito de la Mujer, Editorial Mensajero Bilbao, 1971.

PENA MOLINA, Blanca Olivia: ¿Igualdad o diferencia? Derechos políticos de la mujer y cuota de género en México: estudio de caso en Baja California Sur, Ediciones Congreso del Estado de Baja California Sur-Universidad Autónoma de Baja California Sur, Plaza y Valdés Editores, México, 2003.

RENDÓN GAN, Teresa: Trabajo de Hombres y Trabajo de Mujeres en el México del siglo XX, 2. ${ }^{\text {a }}$ edición, Editorial UNAM, Programa Universitario de Estudios de Género, México, 2006.

RUIZ Celia-DASHA-HIERRO, Graciela: Tres Temas, tres mujeres, muchas mujeres, Editorial UANL, Facultad de Filosofía y Letras, México, 2005. 
TUÑON, Enriqueta: Por fin, ya podemos elegir y ser electas, $1 .^{\mathrm{a}}$ edición, Editorial CONACULTA INAH, México, 2007.

VIDALES-ELIZONDO-RODRÍGUEZ: La Perspectiva de género, breve estudio en Nuevo León, Editorial CECyTE, México, 2007.

WALLECH SCOTT, Joan: Género e Historia, Editorial Fondo de Cultura Económica, México, 2008.

WALLSTEN ALMEGARD, Adrienne Elisabet: Derechos humanos de la mujer en las Conferencias Internacionales: ¿cómo se traducen los derechos humanos de la mujer del plano internacional al plano nacional? el ejemplo de México, Editorial Universidad Iberoamericana, México, 2004.

WASHINGTON VALDEZ, Diana: Cosecha de Mujeres, Safari en el desierto mexicano, Editorial Océano, México, 2005.

ZAPATA CHOISEUL, Margarita: Nuestra Agenda. Consideraciones y Perspectivas en Centroamérica. 1. ${ }^{a}$ edición, Editorial SER, México, 2005.

ZAREMBERG, Griselda: Mujeres, Votos y asistencia social, 1. ${ }^{\mathrm{a}}$ edición, Editorial FLASCO México, México, 2007.

REVISTA GÉNERO: ¿Las mujeres están en la historia?, Asociación colimense Universitaria de Colima, Centro Universitario de Estudios de Género. 